\begin{tabular}{|c|l|}
\hline Title & ELLIPTIC ESTIMA TES INDEPENDENT OF DOMA IN EXPANSION \\
\hline Author(s) & Cho, Yonggeun; Ozawa, Tohru; Shim, Yong-Sun \\
\hline Citation & Hokkaido University Preprint Series in Mathematics, 876, 1-20 \\
\hline Issue Date & 2007 \\
\hline DOI & 10.14943/84026 \\
\hline Doc URL & http://hdl.handle.net/2115/69685 \\
\hline Type & bulletin (article) \\
\hline File Information & pre876.pdf \\
\hline
\end{tabular}

Instructions for use 


\title{
ELLIPTIC ESTIMATES INDEPENDENT OF DOMAIN EXPANSION
}

\author{
YONGGEUN CHO, TOHRU OZAWA AND YONG-SUN SHIM
}

\begin{abstract}
In this paper, we consider elliptic estimates for a system with smooth variable coefficients on a domain $\Omega \subset \mathbb{R}^{n}, n \geq 2$ containing the origin. We first show the invariance of the estimates under a domain expansion defined by the scale that $y=R x, x, y \in \mathbb{R}^{n}$ with parameter $R>1$, provided that the coefficients are in a homogeneous Sobolev space. Then we apply these invariant estimates to the global existence of unique strong solutions to a parabolic system defined on an unbounded domain.
\end{abstract}

\section{INTRODUCTION}

We consider the linear elliptic system $L u=f$ defined by

$$
(L u)^{\alpha} \equiv-\sum_{i, j, \beta} \partial_{i}\left(A_{i j}^{\alpha \beta} \partial_{j} u^{\beta}\right)+m u^{\alpha}=f^{\alpha} \quad \text { in } \quad \Omega_{R} .
$$

Here $u$ is an $N$ dimensional vector field defined on $\mathbb{R}^{n}$ for $N \geq 1, n \geq 2$ and $1 \leq i, j \leq n, 1 \leq \alpha, \beta \leq N . m$ is a nonnegative bounded scalar function. $\Omega_{R}$ is the domain scaled with parameter $R$ and is defined by

$$
\Omega_{R}=\{y: y=R x, \quad x \in \Omega\}, \quad R>1 .
$$

The domain $\Omega$ is a bounded domain with $C^{1,1}$ boundary containing the origin in $\mathbb{R}^{n}$. We assume that the coefficients $A_{i j}^{\alpha \beta}$ and $m$ are functions of $x \in \Omega_{R}$ satisfying the following assumptions:

- $A_{i j}^{\alpha \beta}$ are uniformly continuous on $\overline{\Omega_{R}}, m$ is bounded and for some fixed positive constant $\Lambda$

$$
\sum_{i, j, \alpha, \beta} \frac{\sup }{\Omega_{R}}\left|A_{i j}^{\alpha \beta}\right|+\sup _{\overline{\Omega_{R}}} m \leq \Lambda,
$$

- For some fixed positive constant $\lambda$

$$
\sum_{i, j, \alpha, \beta} A_{i j}^{\alpha \beta}(x) \xi_{i}^{\alpha} \xi_{j}^{\beta} \geq \lambda|\xi|^{2} \quad \text { for all } \quad x \in \Omega_{R}, \quad \xi \in \mathbb{R}^{n N} .
$$

The gradient $\nabla$ is defined by $\nabla=\left(\partial_{1}, \cdots, \partial_{n}\right)$, where $\partial_{i}=\frac{\partial}{\partial x_{i}}$. 
Our main concerns are to find some conditions for elliptic estimates of the operator $L$ independent of domain expansion, namely, independent of the parameter $R>1$.

The elliptic estimate in this paper is the following: if $\Omega$ is a bounded $C^{1,1}$ domain, $\nabla A \in L^{\infty}(\Omega), f \in L^{q}(\Omega)$ and $u$ is a solution of (1.1) in $W_{0}^{1, q} \cap W^{2, q}(\Omega)$ for some $1<q<\infty$, then $u$ satisfies that

$$
\left\|\nabla^{2} u\right\|_{L^{q}(\Omega)} \leq C\left(\|f\|_{L^{q}(\Omega)}+\|u\|_{L^{q}(\Omega)}\right)
$$

where the constant $C$ depends on $\Lambda, \lambda, n, q, \Omega, \partial \Omega,\|\nabla A\|_{L^{\infty}(\Omega)}$, the modulus of continuity of $A$ and so on. Furthermore, if the uniqueness of solutions to the system (1.1) is guaranteed, then we can say that there exists a constant $C$ independent of $u$ and $f$ such that

$$
\left\|\nabla^{2} u\right\|_{L^{q}(\Omega)} \leq C\|f\|_{L^{q}(\Omega)} .
$$

However, we do not know on which parameters the constant $C$ depends exactly (especially, on the scale parameter $R$ ). For details of the estimates (1.5) and (1.6), we refer the readers to the papers and books of $[3,7,10,11,15,16,17]$.

Throughout this paper, we use the notation for Sobolev space $W^{k, q}(\Omega), k \geq$ $0,1 \leq q \leq \infty$ and $H^{k}(\Omega)=W^{k, 2}(\Omega)$. The space $W_{0}^{k, q}(\Omega)(1 \leq q<\infty)$ is the closure of $C_{0}^{k}(\Omega)$ functions in $W^{k, q}(\Omega)$.

Now let us introduce a new question: "Can we get the estimate

$$
\left\|\nabla^{2} u\right\|_{L^{q}\left(\Omega_{R}\right)} \leq C\left(\|f\|_{L^{q}\left(\Omega_{R}\right)}+\|u\|_{L^{q}\left(\Omega_{R}\right)}\right) \quad \text { or } \quad\left\|\nabla^{2} u\right\|_{L^{q}\left(\Omega_{R}\right)} \leq C\|f\|_{L^{q}\left(\Omega_{R}\right)}
$$

with the constant $C$ independent of $R$ ?" If $A$ and $m$ are constants, then the answer is positive. In fact, let $\widetilde{u}(x)=u(R x)$ for a solution $u$ to $L u=f$ in $\Omega_{R}$. Then $\widetilde{u}$ is also a solution to the system $L \widetilde{u}=\tilde{f}$ in $\Omega$, where $\widetilde{f}(x)=R^{2} f(R x)$. The operator $L$ is not changed under the scaling $x \mapsto R x$. Hence we get from (1.5) and (1.6) the estimates

$$
\left\|\nabla^{2} \widetilde{u}\right\|_{L^{q}(\Omega)} \leq C\left(\|\widetilde{f}\|_{L^{q}(\Omega)}+\|\widetilde{u}\|_{L^{q}(\Omega)}\right) \text { and }\left\|\nabla^{2} \widetilde{u}\right\|_{L^{q}(\Omega)} \leq C\|\widetilde{f}\|_{L^{q}(\Omega)}
$$

with $C$ independent of $R$ and by rescaling

$$
\begin{gathered}
\left\|\nabla^{2} \widetilde{u}\right\|_{L^{q}\left(\Omega_{R}\right)} \leq C\left(\|\widetilde{f}\|_{L^{q}\left(\Omega_{R}\right)}+\|\widetilde{u}\|_{L^{q}\left(\Omega_{R}\right)}\right) \\
\left\|\nabla^{2} u\right\|_{L^{q}\left(\Omega_{R}\right)} \leq C\|f\|_{L^{q}\left(\Omega_{R}\right)}
\end{gathered}
$$

for $R>1$, respectively. However, if $A$ is not constant, then the situation is quite different. For an invariant estimate under domain expansion, we need to scrutinize on which factors the constant $C$ depends and to find some conditions for the invariance. 
In this connection, let us introduce an invariant condition under the domain expansion as follows

- (Scaling condition)

$$
\nabla A_{i j}^{\alpha \beta} \in L^{r}\left(\Omega_{R}\right)
$$

for some $r$ such that $n<r<\infty$ if $q \leq n$ and $q \leq r<\infty$ if $q>n$.

Using the conditions (1.3), (1.4), (1.8), we have

Theorem 1.1. Let $\Omega$ be a bounded domain containing the origin of $\mathbb{R}^{n}, n \geq 2$, with $C^{1,1}$ boundary $\partial \Omega$. Assume that $f \in L^{q}\left(\Omega_{R}\right)$ for some $2 \leq q<\infty, A$ and $m$ satisfy the conditions (1.3), (1.4), (1.8) for some fixed $r$ on the scaled domain $\Omega_{R}$. Then there exists a unique solution $u \in\left(W_{0}^{1, q} \cap W^{2, q}\right)\left(\Omega_{R}\right)$ to the boundary value problem $L u=f$ in $\Omega_{R}, R>1, u=0$ on $\partial \Omega_{R}$ satisfying

$$
\|u\|_{W^{2, q}\left(\Omega_{R}\right)} \leq C\left(\|f\|_{L^{q}\left(\Omega_{R}\right)}+\left(1+\|\nabla A\|_{L^{r}\left(\Omega_{R}\right)}\right)^{\frac{2 r}{r-n}}\|u\|_{L^{q}\left(\Omega_{R}\right)}\right) .
$$

The constant $C$ may depend on $\Lambda, \lambda, N, n, q, r, \Omega$ and the modulus continuity of $A$ but not on $R$.

For the proof of Theorem 1.1, we use the classical method of freezing coefficient. Before freezing, we scale the space variables with the factor $R$. Then in freezing coefficients we partition the domain $\Omega$ into $\frac{\rho}{R}$ scaled balls, where $\rho$ is the modulus of continuity of $A$. We revisit the Calderón-Zygmund theories for the whole and half spaces.

If $1<p<2$ and $N \geq 2$, then contrary to the scalar case we cannot assert the uniqueness of solution due to the absence of mean value property nor even the existence of solution to the boundary value problem. However, once $u \in\left(W_{0}^{1, q} \cap\right.$ $\left.W^{2, q}\right)\left(\Omega_{R}\right)$ is a solution of (1.1) for $1<q<2$, the same arguments as above show that $u$ satisfies the estimate (1.9).

Strictly speaking, the right hand side of the estimate (1.9) is not completely uniform on the parameter $R$ because we still do not know how to control the norm $\|u\|_{L^{q}\left(\Omega_{R}\right)}$. Instead if we assume a further condition on $f$ and restrict the range of $q$, then there is a possibility to control it.

To expedite, we introduce some function spaces. We denote by $D^{-1}\left(\Omega_{R}\right)$ the dual space of $D_{0}^{1}\left(\Omega_{R}\right)$ with $<\cdot, \cdot>$ being the dual paring of $D^{-1}\left(\Omega_{R}\right)$ and $D_{0}^{1}\left(\Omega_{R}\right)$. For $n \geq 3$ the space $D_{0}^{1}\left(\Omega_{R}\right)$ is defined by

$$
D_{0}^{1}\left(\Omega_{R}\right)=\left\{v \in L^{\frac{2 n}{n-2}}\left(\Omega_{R}\right):\|v\|_{D_{0}^{1}\left(\Omega_{R}\right)}=\|\nabla v\|_{L^{2}\left(\Omega_{R}\right)}<\infty, v=0 \text { on } \partial \Omega_{R}\right\} .
$$

Indeed, $H_{0}^{1}\left(\Omega_{R}\right)=\left(D_{0}^{1} \cap L^{2}\right)\left(\Omega_{R}\right)$ and $D_{0}^{1}\left(\Omega_{R}\right)=H_{0}^{1}\left(\Omega_{R}\right)$, provided that $\Omega$ is bounded. 
Now if we assume that $f \in\left(D^{-1} \cap L^{q}\right)\left(\Omega_{R}\right)$ and the size of norms is uniform on the parameter $R>1$, then we can remove the term $\|u\|_{L^{q}\left(\Omega_{R}\right)}$ on the right hand side of (1.9) through a scaling invariant estimate, see Lemma 2.4 below.

Theorem 1.2. Suppose that $A, m, f$ and $\Omega$ satisfy the same condition as in Theorem 1.1 with $q, r$ and $n$ satisfying that

$$
\begin{gathered}
q_{k} \leq q<q_{k+1} \text { for } q_{k}<\infty \\
\max (q, n) \leq r<\infty, \quad r \neq n, \quad k \geq 0, \quad n \geq 3,
\end{gathered}
$$

where $q_{k}=\frac{2 n}{n-(4 k+2)}$ if $n>4 k+2, q_{k}=\infty$ by convention if $n \leq 4 k+2$.

If we further assume that $f \in\left(D^{-1} \cap L^{q_{0}}\right)\left(\Omega_{R}\right)$, then the solution $u$ to the system (1.1) satisfies that

$$
\|\nabla u\|_{L^{2}\left(\Omega_{R}\right)} \leq C\|f\|_{D^{-1}\left(\Omega_{R}\right)}
$$

and that

$$
\|u\|_{W^{2, q}\left(\Omega_{R}\right)} \leq C\|f\|_{\left(D^{-1} \cap L^{\left.q_{0} \cap L^{q}\right)\left(\Omega_{R}\right)}\right.}\left(1+\|\nabla A\|_{L^{r}\left(\Omega_{R}\right)}\right)^{\frac{2 r(k+1+\delta)}{r-n}},
$$

where $\delta=\frac{n}{2}\left(1 / q_{k}-1 / q\right)$. Here the constant $C$ also depends on $m$.

If we assume that $\inf _{\Omega_{R}} m \geq \underline{m}$ for some positive constant $\underline{m}$ and $f \in\left(D^{-1} \cap\right.$ $\left.L^{q}\right)\left(\Omega_{R}\right)$ for $2 \leq q<q_{0}$ and $n \geq 2$, then

$$
\begin{gathered}
\|u\|_{H^{1}\left(\Omega_{R}\right)} \leq C\|f\|_{D^{-1}\left(\Omega_{R}\right)}, \\
\|u\|_{W^{2, q}\left(\Omega_{R}\right)} \leq C\|f\|_{\left(D^{-1} \cap L^{q}\right)\left(\Omega_{R}\right)}\left(1+\|\nabla A\|_{L^{r}}\right)^{\frac{2 r}{r-n}} .
\end{gathered}
$$

Remark 1. Note that the 2-dimensional case is included for the second result.

For the estimate of the range of $q$ below $\frac{2 n}{n-2}$, in general it seems very difficult to avoid the assumption $m>\underline{m}$. To do so, we need another condition on $A$. For simplicity, let us assume that $m=0$ and consider the coefficient $A$ of the type $a B+\varepsilon E$. Here $a$ is a scalar $C^{1}$ function with $\nabla a \in L^{r}\left(\Omega_{R}\right)$ for

$$
\max (q, n) \leq r<\infty, \quad r \neq n
$$

and satisfying that $\lambda \leq a \leq \Lambda$ on $\Omega_{R}, B$ is a constant coefficient with $|B| \leq 1$ satisfying (1.4), $\varepsilon$ is a small positive constant and $E$ is in $C^{1}\left(\overline{\Omega_{R}}\right)$ with $\nabla E \in L^{r}\left(\Omega_{R}\right)$ and $|E| \leq \Lambda$ on $\Omega_{R}$. Let us denote the elliptic operators corresponding to the coefficients $A, B$ and $E$ by $L, L_{B}$ and $L_{E}$, respectively. Such $L$ is said to be a small perturbation of the constant type elliptic operator $L_{B}$. 
Theorem 1.3. Let $\Omega$ be a bounded $C^{1,1}$ domain of $\mathbb{R}^{n}, n \geq 2$. If $L$ is a small perturbation of $L_{B}$ and $f \in\left(D^{-1} \cap L^{2} \cap L^{q}\right)\left(\Omega_{R}\right)$ for $2 \leq q<q_{0}=\frac{2 n}{n-2}$, then the boundary value problem $L u=f$ in $\Omega_{R}$ and $u=0$ on $\partial \Omega_{R}$ has a unique solution $u \in\left(W_{0}^{1, q} \cap W^{2, q}\right)\left(\Omega_{R}\right)$ such that $\|\nabla u\|_{L^{2}\left(\Omega_{R}\right)} \leq C\|f\|_{D^{-1}\left(\Omega_{R}\right)}$ and

$$
\|\nabla u\|_{W^{1, q}\left(\Omega_{R}\right)} \leq C\|f\|_{\left(D^{-1} \cap L^{2} \cap L^{q}\right)\left(\Omega_{R}\right)}\left(1+\|\nabla a\|_{L^{r}\left(\Omega_{R}\right)}+\|\nabla E\|_{L^{r}\left(\Omega_{R}\right)}\right)^{\frac{2 r}{r-n}} .
$$

Remark 2. A typical example of the small perturbation of constant type elliptic operator is the Lamé operator $L$, which has variable coefficients $A_{i j}^{\alpha \beta}$ defined by

$$
A_{i j}^{\alpha \beta}(x)=\mu_{1}(x) \delta_{\alpha, \beta} \delta_{i, j}+\left(\mu_{1}+\mu_{2}\right)(x) \delta_{\alpha, i} \delta_{\beta, j}
$$

and hence $L u=-\operatorname{div}\left(\mu_{1} \nabla u\right)-\nabla\left(\mu_{2} \operatorname{div} u\right)$, where $\mu_{1}$ and $\mu_{2}$ are viscosity coefficients satisfying the relation $\mu_{1}(x)=\nu_{1} a(x)+\varepsilon e_{1}(x)$ and $\left(\mu_{1}+\mu_{2}\right)(x)=\nu_{2} a(x)+\varepsilon e_{2}(x)$. This relation is a generalization of the Stokes relation (for instance, see [13]). We assume that $a$ is a $C^{1}$ scalar function with $\lambda \leq a \leq \Lambda$ and $\nabla a \in L^{r}, \nu_{i}$ are fixed positive constants less than 1 and $e_{i}$ is a $C^{1}$ scalar function with $\sum_{i}\left|e_{i}\right| \leq \Lambda$ and $\nabla e_{i} \in L^{r}$. The coefficient $A$ can be rewritten as $A=a B+\varepsilon E$, where

$$
\begin{aligned}
& B_{i j}^{\alpha \beta}=\nu_{1} \delta_{\alpha, \beta} \delta_{i, j}+\nu_{2} \delta_{\alpha, i} \delta_{\beta, j} \\
& E_{i j}^{\alpha \beta}=e_{1} \delta_{\alpha, \beta} \delta_{i, j}+e_{2} \delta_{\alpha, i} \delta_{\beta, j} .
\end{aligned}
$$

The elliptic estimate for Lamé operator $L$ as in Theorem 1.3 can be applied to the heat-conducting compressible Navier-Stokes equations with variable coefficients on an unbounded domain, whole or half space, or exterior domain. One can adapt the same arguments of domain expansion of $[4,5,6]$ to prove the unique solvability of strong solutions.

Remark 3. If $\Omega$ is an exterior domain with compact complement, then we denote the domain $\Omega \cap B_{R}$ by $\widetilde{\Omega}_{R}$ for sufficiently large $R$ so that the complement of $\Omega$ is contained in $B_{R}$. If $\Omega$ is the whole space, then $\widetilde{\Omega}_{R}$ is $B_{R}$. If $\Omega$ is the half space $\mathbb{R}_{+}^{n}$, then we also denote $\widetilde{\Omega}_{R}$ by $R$-scaled domain of $\Omega_{+}$which is a smooth domain satisfies that $\mathbb{R}_{+}^{n} \cap B_{1} \subset \Omega_{+} \subset \Omega \cap B_{2}$. Then a solution $u \in\left(W_{0}^{1, q} \cap W^{2, q}\right)\left(\widetilde{\Omega}_{R}\right)$ of the system (1.1) on $\widetilde{\Omega}_{R}$ satisfies the estimates (1.9), (1.11), (1.12) and (1.13) with $C$ independent of $R>1$.

A direct application of Theorem 1.2 and Theorem 1.3 is to show the elliptic estimates on an unbounded domain $\Omega$. To do this we introduce the homogeneous Sobolev space (for instance see [9])

$$
\begin{gathered}
D^{k, r}(\Omega) \equiv\left\{v \in L_{l o c}^{1}(\Omega):\|v\|_{D^{k, r}(\Omega)}<\infty\right\}, \\
D^{k}(\Omega)=D^{k, 2}(\Omega), \quad\|v\|_{D^{k, r}(\Omega)}=\left\|\nabla^{k} v\right\|_{L^{r}(\Omega)} .
\end{gathered}
$$


Theorem 1.4. Assume that $\Omega$ is an unbounded domain, a whole or half space, or an exterior domain with $C^{1,1}$ boundary and compact complement. Let $f \in\left(D^{-1} \cap\right.$ $\left.L^{q_{0}} \cap L^{q}\right)(\Omega)$ for the same indices $q_{0}, q$ as defined in (1.10) and $A$ be an elliptic coefficient satisfying (1.3), (1.4) and (1.8) .

(1) Then there exists a unique solution $u \in D_{0}^{1} \cap D^{2, q}$ of the elliptic system $L u=f$ with the boundary conditions that $u=0$ on $\partial \Omega$ and $u(x) \rightarrow 0$ as $|x| \rightarrow \infty$. Furthermore, $u$ satisfies that

$$
\begin{gathered}
\|\nabla u\|_{L^{2}(\Omega)} \leq C\|f\|_{D^{-1}(\Omega)}, \\
\|u\|_{W^{2, q}(\Omega)} \leq C\|f\|_{\left(D^{-1} \cap L^{\left.q_{0} \cap L^{q}\right)(\Omega)}\right.}\left(1+\|\nabla A\|_{L^{r}(\Omega)}\right)^{\frac{2 r(k+1+\delta)}{r-n}} .
\end{gathered}
$$

(2) If we further assume that $m \geq \underline{m}$ for some positive constant $\underline{m}, 2 \leq q<q_{0}$ and $n \geq 2$, then

$$
\begin{gathered}
\|u\|_{H_{0}^{1}(\Omega)} \leq C\|f\|_{D^{-1}(\Omega)}, \\
\|u\|_{W^{2, q}(\Omega)} \leq C\|f\|_{\left(D^{-1} \cap L^{q}\right)(\Omega)}\left(1+\|\nabla A\|_{L^{r}}\right)^{\frac{2 r}{r-n}} .
\end{gathered}
$$

(3) If $L$ is a small perturbation of $L_{B}$ as in Theorem 1.3 and $f \in\left(D^{-1} \cap L^{2} \cap\right.$ $\left.L^{q}\right)(\Omega)$ for $2 \leq q<q_{0}$, then

$$
\|\nabla u\|_{W^{1, q}(\Omega)} \leq C\|f\|_{\left(D^{-1} \cap L^{2} \cap L^{q}\right)(\Omega)}\left(1+\|\nabla a\|_{L^{r}(\Omega)}+\|\nabla E\|_{L^{r}(\Omega)}\right)^{\frac{2 r}{r-n}} .
$$

Secondly, we apply the above theorems to a linear parabolic system:

$$
\begin{aligned}
\varphi u_{t}+L u & =F \text { in }[0, T] \times \Omega, \quad u(0, x)=u_{0}(x) \text { in } \Omega, \\
(L u)^{\alpha} & =-\sum_{i, j, \beta} \partial_{i}\left(A_{i j}^{\alpha \beta} \partial_{j} u^{\beta}\right) .
\end{aligned}
$$

Here $\varphi$ is assumed to be a nonnegative function which has a compact support or decays at space infinity. For the simplicity of presentation we only consider that the domain $\Omega$ is the whole $\mathbb{R}^{3}$ or half $\mathbb{R}_{+}^{3}$ space, or an exterior domain of $\mathbb{R}^{3}$ with $C^{1,1}$ boundary and compact complement. $A_{i j}^{\alpha \beta}$ is $C_{b}^{1}([0, T] \times \Omega)$ satisfying the conditions (1.3), (1.4), and the symmetry condition

$$
A_{j i}^{\beta \alpha}=A_{i j}^{\alpha \beta} \text { for all } i, j, \alpha, \beta .
$$

Since $\varphi$ can vanish on some open set or decay at space infinity, it is difficult to expect the uniqueness and regularity of solutions. For this purpose, we assume the following compatibility condition that for any $v \in C_{0}^{1}(\Omega)$

$$
\sum_{i, j, \alpha, \beta} \int_{\Omega} A_{i j}^{\alpha \beta}(0, x) \partial_{j} u_{0}^{\beta} \partial_{i} v^{\alpha} d x=\sum_{\alpha} \int_{\Omega}\left(F(0)^{\alpha}-\sqrt{\varphi} g^{\alpha}\right) v^{\alpha} d x
$$

for some $g=\left(g^{\alpha}\right) \in L^{2}(\Omega)$. 
Theorem 1.5. Assume that

$$
\begin{gathered}
0 \leq \varphi \in\left(L^{\frac{3}{2}} \cap L^{\infty}\right)(\Omega), u_{0} \in D_{0}^{1}(\Omega), \\
F, F_{t} \in L^{2}\left(0, T ; D^{-1}(\Omega)\right), F \in L^{2}\left(0, T ; L^{6}(\Omega)\right)
\end{gathered}
$$

and also assume that $A=A(t, x) \in C_{b}^{1}([0, T] \times \Omega)$ satisfies (1.3), (1.4) and (1.18) for each $t$. If we further assume the compatibility condition (1.19), then there exists a unique solution $u \in L^{\infty}\left(0, T_{*} ; D_{0}^{1}(\Omega)\right)$ such that $\nabla u \in L^{2}\left(0, T ; W^{1,6}(\Omega)\right)$ and $u_{t} \in L^{2}\left(0, T ; D_{0}^{1}(\Omega)\right)$.

For the proof we localize the problem and consider the case where $\varphi$ has a positive lower bound. Then we show that the localized solutions satisfy a priori estimates uniform for the domain expansion.

\section{Preliminary Lemmas}

Before proving the theorems, let us introduce some lemmas which are crucial for the proof of the theorems. The first one is on the elliptic regularity in the case of constant coefficients.

Lemma 2.1. Let $A$ be a constant coefficient satisfying (1.3) and (1.4), and let $v$ be a vector field in $D^{2}\left(\mathbb{R}^{n}\right)$. Then for any $1<q<\infty$ there exists constant $C$ depend only on $\lambda, \Lambda, N, n, q$ such that

$$
\left\|\nabla^{2} v\right\|_{L^{q}} \leq C\left\|L_{0} v\right\|_{L^{q}}
$$

where

$$
\left(L_{0} v\right)^{\alpha}=-\sum_{i, j, \beta} \partial_{i}\left(A_{i j}^{\alpha \beta} \partial_{j} u^{\beta}\right) .
$$

Proof of Lemma 2.1. One may find a proof using fundamental solution to the equation $L_{0} v=0$ in $\mathbb{R}^{n}$ in $[2,3]$. Here we introduce a simplified version for a second order elliptic operator.

By density, we may assume that $u \in C_{0}^{2}$. Using Fourier transform such that $\widehat{g}(\xi)=\int e^{-i x \cdot \xi} g(x) d x$, we have

$$
\widehat{\left(L_{0} v\right)^{\alpha}}(\xi)=-\sum_{i, j, \beta} A_{i j}^{\alpha \beta} \xi_{i} \xi_{j} \widehat{v^{\beta}}(\xi)=-\sum_{i, j, \beta} A_{i j}^{\alpha \beta} \xi_{i}^{\prime} \xi_{j}^{\prime}|\xi|^{2} \widehat{v^{\beta}}(\xi) \equiv \sum_{\beta} M^{\alpha \beta}\left(\xi^{\prime}\right) \widehat{\Delta v^{\beta}},
$$

where

$$
M^{\alpha \beta}\left(\xi^{\prime}\right)=\sum_{i, j} A_{i j}^{\alpha \beta} \xi_{i}^{\prime} \xi_{j}^{\prime} \quad \text { and } \quad \xi_{j}^{\prime}=\frac{\xi_{j}}{|\xi|}
$$


From the strong ellipticity (1.4), we deduce that

$$
\begin{gathered}
\sum_{\alpha, \beta} M^{\alpha \beta}\left(\xi^{\prime}\right) \eta_{\alpha} \eta_{\beta} \geq \lambda|\eta|^{2} \quad \text { for all } \eta \in \mathbb{R}^{N}, \\
|\operatorname{det} M| \geq C(N) \lambda^{N}, \\
\left|\nabla^{k} M^{\alpha \beta}\right| \leq C(N, n, k) \Lambda|\xi|^{-k} \quad \text { for all } \quad k \geq 0 .
\end{gathered}
$$

Thus using the recursion

$$
\nabla M^{-1}=-M^{-1}(\nabla M) M^{-1} \quad \text { and } \quad \nabla^{k} M^{-1}=-\nabla^{k-1}\left(M^{-1}(\nabla M) M^{-1}\right),
$$

we obtain

$$
\left|\nabla^{k} M^{-1}\right| \leq C \Lambda^{-1}\left(\frac{\Lambda}{\lambda}\right)^{(k+1) N}|\xi|^{-k} \text { for all } \xi \neq 0 \text { and } \quad k \geq 0
$$

From (2.2), we have

$$
\widehat{\Delta v^{\alpha}}(\xi)=\sum_{\beta}\left(M^{-1}\right)^{\alpha \beta} \widehat{L_{0} v^{\beta}}(\xi)
$$

and hence from (2.3) we deduce that by the Fourier multiplier theorem (for instance, see Proposition 2, p. 245 of [18]), (2.1) holds.

The following is a half-space version of Lemma 2.1 (for the proof of scalar case see $[11])$.

Lemma 2.2. Let $A$ be a constant coefficient satisfying (1.3) and (1.4), and let $v$ be a vector field in $W_{0}^{1,1}\left(\Omega^{+}\right)$and be a weak solution of $L_{0} v=f$ in $\Omega^{+}$with $u=0$ near $(\partial \Omega)^{+}$for some $f \in L^{q}$ with $1<q<\infty$, where $\Omega^{+}=\Omega \cap \mathbb{R}_{+}^{n}$ and $(\partial \Omega)^{+}=\partial \Omega \cap \mathbb{R}_{+}^{n}$ for some bounded domain $\Omega$ containing the origin and having $C^{1,1}$ boundary. Then $v \in\left(W_{0}^{q} \cap W^{2, q}\right)\left(\Omega^{+}\right)$and there exists a constant $C$ depending only on $\lambda, \Lambda, N, n, q$ such that

$$
\left\|\nabla^{2} v\right\|_{L^{q}\left(\Omega^{+}\right)} \leq C\left\|L_{0} v\right\|_{L^{q}\left(\Omega^{+}\right)} .
$$

Proof of Lemma 2.2. We extend $v$ and $f$ to $\mathbb{R}_{+}^{n}$ by defining zero outside of $\Omega^{+}$. Let the extended functions be still $v$ and $f$. Then by the support condition $v$ is clearly a weak solution of $L_{0} v=f$ in $\mathbb{R}_{+}^{n}$. Hence by the representation of solution in half space by the fundamental solution $[2,3]$, we conclude that $v \in W^{2, q}\left(\mathbb{R}_{+}^{n}\right)$ and $(2.4)$ holds.

Furthermore, since $v^{\alpha}$ is $W^{2, q}\left(\Omega^{+}\right)$function for each $\alpha$, there are $N$ functions $g^{\alpha} \in L^{q}$ such that $\Delta v^{\alpha}=g^{\alpha}$ in $\Omega^{+}$. Hence by setting $v^{\alpha}=g^{\alpha}=0$ outside of $\Omega^{+}$and extending $v^{\alpha}$ and $g^{\alpha}$ via odd reflection to $\mathbb{R}^{n}$ and using a compactly supported even smooth function $\eta$, one can show that $v^{\alpha} * \eta_{\delta} \rightarrow v^{\alpha}$ in $W^{2, q}$ as $\delta \rightarrow 0$ and $\left(v^{\alpha} * \eta_{\delta}\right)\left(x^{\prime}, 0\right)=0$, where $*$ denotes the convolution and $\eta_{\delta}(x)=$ 
$\delta^{-n} \eta(x / \delta)$. Therefore we conclude that $v \in W_{0}^{1, q}\left(\Omega_{+}\right)$. This completes the proof of the lemma.

The third one is on the regularity of solutions of (1.1).

Lemma 2.3. Let $u \in\left(W_{0}^{1, q} \cap W^{2, q}\right)(\Omega)$ be a solution to the system (1.1) on a bounded $C^{1,1}$-domain $\Omega$ with $A$ satisfying (1.3), (1.4) and (1.8) for some $1<q<$ $\infty$. Then if $f \in L^{s}(\Omega)$ for some $s$ with $q<s<\infty$, then $u \in\left(W_{0}^{1, s} \cap W^{2, s}\right)(\Omega)$.

Proof of Lemma 2.3. By applying Caledrón-Zygmund theory equipped with Lemmas 2.1 and 2.2 to the local problems over the interior and near the boundary of $\Omega$, the regularity result follows from the contraction argument in $L^{p}$ theory of [14]. See also the proof of Lemma 9.16 in [11]. We leave the details of proof to the readers.

Finally, we introduce some Sobolev inequalities invariant under domain expansion.

Lemma 2.4. Assume that $\Gamma$ is a $C^{1,1}$ domain in $\mathbb{R}^{n}, n \geq 2$ and $v \in W^{k, s}(\Gamma)$. Let $\ell$ be a number such that $s \leq \ell \leq \frac{s n}{n-k s}$ if $k s<n, s \leq \ell \leq \infty$ if $k s>n$ or $s \leq \ell<\infty$ if $k s=n$. Then there exists a constant $C$ depending on $\Gamma, \ell, n, k, s$ such that for any $R>1$

$$
\|v\|_{L^{\ell}\left(\Gamma_{R}\right)} \leq C\|v\|_{L^{s}\left(\Gamma_{R}\right)}^{1-\delta}\|v\|_{W^{k, s}\left(\Gamma_{R}\right)}^{\delta},
$$

where $\delta=\frac{n}{k}(1 / s-1 / \ell)$ and $\Gamma_{R}$ is the $R$-scaled domain of $\Gamma$ defined by (1.2).

Proof of Lemma 2.4. For the proof of (2.5) with $R=1$, see the proof of Theorem 5.8 of [1]. For the invariance, let $v(x)=w(R x)$. Then by change of variables, we have for any $R>1$

$$
R^{-\frac{n}{\ell}}\|w\|_{L^{\ell}\left(\Gamma_{R}\right)} \leq C R^{-\frac{n}{s}(1-\delta)}\|w\|_{L^{s}\left(\Gamma_{R}\right)}^{1-\delta} R^{\left(k-\frac{n}{s}\right) \delta}\|w\|_{W^{k, s}\left(\Gamma_{R}\right)}^{\delta} .
$$

Hence the scaling invariance follows from the fact $k \delta=n(1 / s-1 / \ell)$.

\section{Independence of Domain expansion; Proof of Theorem 1.1}

In this section, we prove Theorem 1.1. If $q=2$, then since the operator $L$ is of divergence form, the existence and uniqueness of solution $u \in\left(H_{0}^{1} \cap H^{2}\right)\left(\Omega_{R}\right)$ readily follows from the Lax-Milgram theorem and the standard method of difference quotient. We refer the readers to the book [10]. If $q>2$, then since the domain is bounded, the existence and the uniqueness follow automatically from the $L^{2}$ theory and Lemma 2.3. Thus we have only to consider the estimates (1.9) and (1.11). 
For the proof, we scale functions by a parameter $R$ and define the scaled functions by $\widetilde{u}(x)=u(R x), \widetilde{A}(x)=A(R x), \widetilde{m}(x)=R^{2} m(R x)$ and $\widetilde{f}(x)=R^{2} f(R x)$. Also we define a scaled operator $\widetilde{L}$ by

$$
(\widetilde{L} \widetilde{u})^{\alpha} \equiv-\sum_{i, j, \beta} \partial_{i}\left(\widetilde{A}_{i j}^{\alpha \beta} \widetilde{u}^{\beta}\right)+\widetilde{m} \widetilde{u}^{\alpha}=\widetilde{f}^{\alpha} \quad \text { on } \quad \Omega .
$$

Now fixing $R>1$, we freeze the coefficient $\widetilde{A}$ near the point $x_{0} \in \Omega^{\prime}$, where $\Omega^{\prime}$ is a precompact subset of $\Omega$ (for example we can take $\Omega^{\prime}=\Omega \backslash\left(B\left(0, \varepsilon_{0}\right)+\partial \Omega\right.$ ) for small $\varepsilon_{0}$, where $\varepsilon_{0}$ depends only on $\rho$, the modulus of continuity of $A$ ). Let $L_{0}$ be the constant coefficient operator given by $L_{0} v=\widetilde{A}\left(x_{0}\right) \nabla^{2} v$. Suppose $v$ has support in a ball $B\left(x_{0}, \frac{\rho}{R}\right)$ with $\rho<\frac{1}{2} \operatorname{dist}\left(\partial \Omega, \Omega^{\prime}\right)$ (hereafter we denote by $B_{\frac{\rho}{R}}$ the ball $\left.B\left(x_{0}, \frac{\rho}{R}\right)\right)$. Then we have

$$
L_{0} v=-\left(\widetilde{A}\left(x_{0}\right)-\widetilde{A}\right) \nabla^{2} v-\widetilde{A} \nabla^{2} v,
$$

and by $(2.1)$

$$
\begin{aligned}
\left\|\nabla^{2} v\right\|_{L^{q}\left(B \frac{\rho}{R}\right)} & \leq C\left\|L_{0} v\right\|_{L^{q}\left(B_{\frac{\rho}{R}}\right)} \\
& \left.\leq C \sup _{\frac{\rho}{R}}\left|\widetilde{A}\left(x_{0}\right)-\widetilde{A}\right|\left\|\nabla^{2} v\right\|_{L^{q}\left(B_{\frac{\rho}{R}}\right)}+\left\|\widetilde{A} \nabla^{2} v\right\|_{L^{q}\left(B_{\frac{\rho}{R}}\right)}\right) .
\end{aligned}
$$

Since $\widetilde{A}$ is uniformly continuous on $\overline{\Omega^{\prime}}$, there exists a positive number $\delta$ independent of $R$ such that

$$
\left|\widetilde{A}\left(x_{0}\right)-\widetilde{A}(x)\right| \leq \frac{1}{2 C}
$$

if $\left|x-x_{0}\right|<\frac{\delta}{R}$. Actually we can choose uniform $\delta$ for any $x_{0} \in \Omega^{\prime}$ from the conditions (1.3) and (1.4). Hence we have

$$
\left\|\nabla^{2} v\right\|_{L^{q}\left(B_{\frac{\rho}{R}}\right)} \leq C(n, q, \lambda, \Lambda)\left\|\widetilde{A} \nabla^{2} v\right\|_{L^{q}\left(B \frac{\rho}{R}\right)},
$$

provided $\rho \leq \delta$.

Choose a smooth cutoff function $\eta \in C_{0}^{2}\left(B_{\frac{\rho}{R}}\right)$ such that $\eta=1$ on $B_{\frac{\rho}{2 R}}, \eta=0$ on $B_{\frac{\rho}{R}} \backslash B_{\frac{3}{4} \frac{\rho}{R}}$ and $|\nabla \eta| \leq \frac{8 R}{\rho},\left|\nabla^{2} \eta\right| \leq \frac{64 R^{2}}{\rho^{2}}$. Then $v=\eta \widetilde{u} \in W_{0}^{2, q}(\Omega)$ and we have

$$
\begin{aligned}
& \left\|\nabla^{2} \widetilde{u}\right\|_{L^{q}\left(B_{\frac{\rho}{2 R}}\right)} \leq\left\|\nabla^{2} v\right\|_{L^{q}\left(B_{\frac{\rho}{R}}\right)} \\
& \leq C\left(\left\|\eta \widetilde{A} \nabla^{2} \widetilde{u}\right\|_{L^{q}\left(B_{\frac{\rho}{R}}\right)}+\|\widetilde{A} \nabla \eta \nabla \widetilde{u}\|_{L^{q}\left(B_{\frac{\rho}{R}}\right)}+\left\|A\left(\nabla^{2} \eta\right) \widetilde{u}\right\|_{L^{q}\left(B_{\frac{\rho}{R}}\right)}\right) \\
& \leq C\left(\|\widetilde{f}\|_{L^{q}\left(B_{\frac{3}{4}} \frac{\rho}{R}\right)}+\|\widetilde{m} \widetilde{u}\|_{L^{q}\left(B_{\frac{3}{4}} \frac{\rho}{R}\right)}\right. \\
& \left.\quad \quad+\|\nabla \widetilde{A} \nabla \widetilde{u}\|_{L^{q}\left(B_{\frac{3}{4} \frac{\rho}{R}}\right)}+\|\widetilde{A} \nabla \eta \nabla \widetilde{u}\|_{L^{q}\left(B_{\left.\frac{\rho}{R}\right)}\right.}+\left\|\widetilde{A}\left(\nabla^{2} \eta\right) \widetilde{u}\right\|_{L^{q}\left(B_{\frac{\rho}{R}}\right)}\right) \\
& \quad \leq C\left(\|\widetilde{f}\|_{L^{q}\left(B_{\frac{3}{4} \frac{\rho}{R}}\right)}+\|\nabla \widetilde{A} \nabla \widetilde{u}\|_{L^{q}\left(B_{\frac{3}{4} \frac{\rho}{R}}\right)}+\frac{R}{\rho}\|\nabla \widetilde{u}\|_{L^{q}\left(B_{\frac{3}{4} \frac{\rho}{R}}\right)}+\frac{R^{2}}{\rho^{2}}\|\widetilde{u}\|_{L^{q}\left(B_{\frac{3}{4} \frac{\rho}{R}}\right)}\right),
\end{aligned}
$$

provided $\rho \leq \delta \leq 1$. 
By the Hölder inequality $\|\nabla \widetilde{A} \nabla \widetilde{u}\|_{L^{q}\left(B_{\left.\frac{3}{4} \frac{\rho}{R}\right)}\right.} \leq\|\nabla \widetilde{A}\|_{L^{r}(\Omega)}\|\nabla \widetilde{u}\|_{L^{\frac{r q}{r-q}\left(B_{\frac{3}{4}} \frac{\rho}{R}\right)}}$ (with convention $\frac{r q}{r-q}=\infty$ if $r=q$ ), we have

$$
\begin{array}{r}
\left\|\nabla^{2} \widetilde{u}\right\|_{L^{q}\left(B_{\left.\frac{\rho}{2 R}\right)}\right)} \leq C\left(\|\widetilde{f}\|_{L^{q}\left(B_{\frac{3}{4} \frac{\rho}{R}}\right)}+\|\nabla \widetilde{A}\|_{L^{r}(\Omega)}\|\nabla \widetilde{u}\|_{L^{\frac{r q}{r-q}\left(B_{\frac{3}{4}} \frac{\rho}{R}\right)}}\right. \\
\left.+\frac{R}{\rho}\|\nabla \widetilde{u}\|_{L^{q}\left(B_{\left.\frac{3}{4} \frac{\rho}{R}\right)}\right.}+\frac{R^{2}}{\rho^{2}}\|\widetilde{u}\|_{L^{q}\left(B_{\frac{3}{4} \frac{\rho}{R}}\right)}\right) .
\end{array}
$$

For the estimate $\|\nabla \widetilde{u}\|_{L^{\frac{r q}{r-q}}\left(B_{\frac{3}{4} \frac{\rho}{R}}\right)}$, we apply the Sobolev embedding (2.5) with $s=q, \ell=\frac{r q}{r-q}, k=1$ and $\stackrel{4}{\Gamma}=B_{1}$. Since $q<\frac{r q}{r-q}<\frac{n q}{n-q}$ for $q \leq n$ and $q<\frac{r q}{r-q} \leq \infty$ for $q>n$ (here we used the convention that $\frac{n q}{n-q}=\infty$ if $q \geq n$ ), we deduce that

$$
\begin{aligned}
& \|\nabla \widetilde{u}\|_{L^{\frac{r q}{r-q}}\left(B_{\frac{3}{4}} \frac{\rho}{R}\right)} \\
& \leq C\left(\frac{\rho}{R}\right)^{\frac{n(r-q)}{r q}}\left\|\nabla \widetilde{u}\left(\frac{3}{4} \frac{\rho}{R} \cdot\right)\right\|_{L^{\frac{r q}{r-q}}\left(B_{1}\right)} \\
& \leq C\left(\frac{\rho}{R}\right)^{\frac{n(r-q)}{r q}}\left\|\nabla \widetilde{u}\left(\frac{3}{4} \frac{\rho}{R} \cdot\right)\right\|_{L^{q}\left(B_{1}\right)}^{1-\frac{n}{r}}\left\|\nabla \widetilde{u}\left(\frac{3}{4} \frac{\rho}{R} \cdot\right)\right\|_{W^{1, q}\left(B_{1}\right)}^{\frac{n}{r}} \\
& \leq C\left(\frac{\rho}{R}\right)^{\frac{n(r-q)}{r q}}\left(\left\|\nabla \widetilde{u}\left(\frac{3}{4} \frac{\rho}{R} \cdot\right)\right\|_{L^{q}\left(B_{1}\right)}\right. \\
& \left.+\left(\frac{\rho}{R}\right)^{\frac{n}{r}}\left\|\nabla \widetilde{u}\left(\frac{3}{4} \frac{\rho}{R} \cdot\right)\right\|_{L^{q}\left(B_{1}\right)}^{1-\frac{n}{r}}\left\|\nabla^{2} \widetilde{u}\left(\frac{3}{4} \frac{\rho}{R} \cdot\right)\right\|_{L^{q}\left(B_{1}\right)}^{\frac{n}{r}}\right) \\
& \leq C\left(\frac{\rho}{R}\right)^{\frac{n(r-q)}{r q}}\left(\left(\frac{\rho}{R}\right)^{-\frac{n}{q}}\|\nabla \widetilde{u}\|_{L^{q}\left(B_{\frac{3}{4}} \frac{\rho}{R}\right)}\right. \\
& \left.+\left(\frac{\rho}{R}\right)^{\frac{n}{r}-\frac{n}{q}}\|\nabla \widetilde{u}\|_{L^{q}\left(B_{\left.\frac{3}{4} \frac{\rho}{R}\right)}\right.}^{1-\frac{n}{r}}\left\|\nabla^{2} \widetilde{u}\right\|_{L^{q}\left(B_{\frac{3}{4}} \frac{\rho}{R}\right)}^{\frac{n}{R}}\right) \\
& =C\left(\left(\frac{R}{\rho}\right)^{\frac{n}{r}}\|\nabla \widetilde{u}\|_{L^{q}\left(B_{\frac{3}{4}} \frac{\rho}{R}\right)}+\|\nabla \widetilde{u}\|_{L^{q}\left(\frac{n}{r} \frac{\frac{n}{4}}{R}\right)}^{1-\frac{n}{R}}\left\|\nabla^{2} \widetilde{u}\right\|_{L^{q}\left(B_{\frac{3}{4}} \frac{\rho}{R}\right)}^{\frac{n}{R}}\right),
\end{aligned}
$$

where the constant $C$ does not depend on $\rho$ and $R$.

Substituting (3.4) into (3.3), by Young's inequality we have that for some $\varepsilon>0$ and small $\rho$

$$
\begin{aligned}
& \left\|\nabla^{2} \widetilde{u}\right\|_{L^{q}\left(B_{\frac{\rho}{2 R}}\right)} \\
& \leq C_{\varepsilon}\left(\|\widetilde{f}\|_{L^{q}\left(B_{\left.\frac{3}{4} \frac{\rho}{R}\right)}\right.}+\left(\left(\frac{R}{\rho}\right)^{\frac{n}{r}}\|\nabla \widetilde{A}\|_{L^{r}(\Omega)}+\|\nabla \widetilde{A}\|_{L^{r}(\Omega)}^{\frac{r}{r-n}}\right)\|\nabla \widetilde{u}\|_{L^{q}\left(B_{\frac{3}{4}} \frac{\rho}{R}\right)}\right. \\
& \left.\quad+\frac{R}{\rho}\|\nabla \widetilde{u}\|_{L^{q}\left(B_{\frac{3}{4}} \frac{\rho}{R}\right)}+\frac{R^{2}}{\rho^{2}}\|\widetilde{u}\|_{L^{q}\left(B_{\frac{3}{4}} \frac{\rho}{R}\right)}\right)+\varepsilon\left\|\nabla^{2} \widetilde{u}\right\|_{L^{q}\left(B_{\frac{3}{4}} \frac{\rho}{R}\right)} .
\end{aligned}
$$

Fixing $\rho$, choose a finite covering $\left\{B_{k}^{\prime}\right\}_{k=1}^{K}$ and $\left\{B_{k}\right\}_{k=1}^{K}$ of $\Omega^{\prime}$ such that $B_{k}^{\prime}=$ $B\left(x_{k}, \frac{\rho}{2 R}\right)$ and $B_{k}=B\left(x_{k}, \frac{3}{4} \frac{\rho}{R}\right)$ with $x_{k} \in \Omega^{\prime}, \Omega^{\prime} \subset \cup_{k} B_{k}^{\prime} \subset \Omega$ and $\sum_{k} \chi_{B_{k}}(x) \leq c_{n}$ 
for some fixed positive number $c_{n}$. The number $c_{n}$ can be taken by $2^{n}$ by the finite overlapping property of balls. Hence we have

$$
\begin{aligned}
& \left\|\nabla^{2} \widetilde{u}\right\|_{L^{q}\left(\Omega^{\prime}\right)}^{q} \leq \sum_{k}\left\|\nabla^{2} \widetilde{u}\right\|_{L^{q}\left(B_{k}^{\prime}\right)}^{q} \\
& \leq C_{\varepsilon} \sum_{k}\left(\|\widetilde{f}\|_{L^{q}\left(B_{k}\right)}^{q}+\mathbf{A}(n, r, \rho, R)^{q}\|\nabla \widetilde{u}\|_{L^{q}\left(B_{k}\right)}^{q}\right. \\
& \left.\quad+\frac{R^{q}}{\rho^{q}}\|\nabla \widetilde{u}\|_{L^{q}\left(B_{k}\right)}^{q}+\frac{R^{2 q}}{\rho^{2 q}}\|\widetilde{u}\|_{L^{q}\left(B_{k}\right)}^{q}\right)+\varepsilon^{q} \sum_{k}\left\|\nabla^{2} \widetilde{u}\right\|_{L^{q}\left(B_{k}\right)}^{q} \\
& \leq C_{\varepsilon} c_{n}\left(\|\widetilde{f}\|_{L^{q}(\Omega)}^{q}+\mathbf{A}(n, r, \rho, R)^{q}\|\nabla \widetilde{u}\|_{L^{q}(\Omega)}^{q}\right. \\
& \left.\quad+\frac{R^{q}}{\rho^{q}}\|\nabla \widetilde{u}\|_{L^{q}}^{q}+\frac{R^{2 q}}{\rho^{2 q}}\|\widetilde{u}\|_{L^{q}(\Omega)}^{q}\right)+\varepsilon^{q}\left\|\nabla^{2} \widetilde{u}\right\|_{L^{q}(\Omega)}^{q},
\end{aligned}
$$

where

$$
\mathbf{A}(n, r, \rho, R)=\left(\frac{R}{\rho}\right)^{\frac{n}{r}}\|\nabla \widetilde{A}\|_{L^{r}(\Omega)}+\|\nabla \widetilde{A}\|_{L^{r}(\Omega)}^{\frac{r}{r-n}} .
$$

Now let us denote the $2 \varepsilon_{0}$ neighborhood of the boundary $\partial \Omega$ by $\Omega_{\varepsilon_{0}}$ for some fixed $\varepsilon_{0}$ to be chosen later, that is, $\Omega_{\varepsilon_{0}}=\partial \Omega+B\left(0,2 \varepsilon_{0}\right)$. Then $\Omega \backslash \Omega^{\prime} \subset \Omega_{\varepsilon_{0}}$. Since $\partial \Omega$ is uniformly smooth, there are finite number of points $x_{k} \in \partial \Omega, 1 \leq$ $k \leq N$ with a neighborhood $\mathcal{N}_{k}=\mathcal{N}_{x_{k}}$ containing a ball $B\left(x_{k}, 3 \varepsilon_{0}\right)$ and hence $\Omega_{\varepsilon_{0}} \subset \bigcup_{1 \leq k \leq K^{\prime}} B\left(x_{k}, 3 \varepsilon_{0}\right)$, and with a diffeomorphism $\Psi=\Psi_{k}$ from $\mathcal{N}_{k}$ onto unit ball $B=B(0,1)$ in $\mathbb{R}^{n}$ such that $\Psi_{k}\left(\mathcal{N}_{k} \cap \Omega\right) \subset \mathbb{R}_{+}^{n}, \Psi_{k}\left(\mathcal{N}_{k} \cap \partial \Omega\right) \subset \partial \mathbb{R}_{+}^{n}$. Write $y=\Psi_{k}(x), \mathfrak{u}(y)=\widetilde{u}(x), x \in \mathcal{N}_{k}, y \in B$. Fixing $k$, we denote $\mathcal{N}_{k}$ and $\Psi_{k}$ by $\mathcal{N}$ and $\Psi$, respectively. Then we have

$$
\mathfrak{L} \mathfrak{u}=\mathfrak{f} \quad \text { in } \quad B^{+}=B \cap \mathbb{R}_{+}^{n},
$$

where

$$
\begin{aligned}
& (\mathfrak{L} \mathfrak{u})^{\alpha}=\sum_{\beta, i, j} \mathfrak{A}_{i j}^{\alpha \beta} \partial_{i} \partial_{j} \mathfrak{u}^{\beta}+\sum_{\beta, k} \mathfrak{B}_{k}^{\alpha \beta} \partial_{k} \mathfrak{u}^{\beta}+\mathfrak{m} \mathfrak{u}^{\alpha}, \\
& \mathfrak{f}(y)=\widetilde{f}\left(\Psi^{-1}(y)\right), \quad \mathfrak{m}(y)=\widetilde{m}\left(\Psi^{-1}(y)\right), \\
& \mathfrak{A}_{i j}^{\alpha \beta}(y)=-\sum_{k, l} \widetilde{A}_{k l}^{\alpha \beta}\left(\Psi^{-1}(y)\right) \partial_{k} \Psi_{i}(y) \partial_{l} \Psi_{j}(y) \\
& \mathfrak{B}_{k}^{\alpha \beta}(y)=-\sum_{i, j} \widetilde{A}_{i j}^{\alpha \beta}\left(\Psi^{-1}(y)\right) \partial_{i} \partial_{j} \Psi_{k}(y)-\sum_{i, j, l} \partial_{k} \widetilde{A}_{i j}^{\alpha \beta}\left(\Psi^{-1}(y)\right) \partial_{i} \Psi_{l}(y) \partial_{j} \Psi_{k}(y) .
\end{aligned}
$$

Since the Jacobian matrix $\nabla \Psi$ is invertible, there exists a constant $C(\Psi)$ such that $C(\Psi)^{-1}|x| \leq\left|\nabla \Psi\left(\Psi^{-1}(y)\right) x\right| \leq C(\Psi)|x|$ for all $y \in B$ and any $x \in \mathbb{R}^{n}$. Thus from this we deduce that $\sum_{\alpha, \beta, i, j} \mathfrak{A}_{i j}^{\alpha \beta} \xi_{j}^{\alpha} \xi_{j}^{\beta} \geq \lambda|(\nabla \Psi) \xi|^{2} \geq C(\Psi)^{-2} \lambda|\xi|^{2}$ for any $\xi \in \mathbb{R}^{n N}$ and also that $\mathfrak{A}$ is uniformly continuous on $B_{+}$and has the modulus continuity $\frac{\rho^{*}}{R}$, where $\rho^{*}$ is a small number depending on $\rho$ and $\nabla \Psi$. 
Now we choose a half ball $B_{+}^{\prime}=B(0, s) \cap \mathbb{R}_{+}^{n}$ with $0<s<1$ such that $B\left(x_{k}, 2 \varepsilon_{0}\right) \subset \Psi^{-1}\left(B_{+}^{\prime}\right) \subset B\left(x_{k}, 3 \varepsilon_{0}\right)$. Then we have only to apply the same proof for interior estimate on the domain $\Omega^{\prime}$ to the half ball $B_{+}^{\prime}$. For this purpose, we take $\frac{\rho^{*}}{R}$ scaled balls covers the half ball $B_{+}^{\prime}$ and cut-off functions supported in the balls. For the estimates on the half balls which are half balls of $\frac{\rho^{*}}{R}$ scale covering $B_{+}^{\prime}$ with centers $B(0, s) \cap \partial \mathbb{R}_{+}^{n}$, we use the half space estimate of Lemma 2.2. Actually we have

$$
\begin{aligned}
& \left\|\nabla^{2} \mathfrak{u}\right\|_{L^{q}\left(B_{+}^{\prime}\right)}^{q} \\
& \leq C\left(\|\mathfrak{f}\|_{L^{q}\left(B_{+}\right)}^{q}+\left(\left(\frac{R}{\rho^{*}}\right)^{\frac{n q}{r}}\|\nabla \mathfrak{A}\|_{L^{r}\left(B_{+}\right)}^{q}+\|\nabla \mathfrak{A}\|_{L^{r}\left(B_{+}\right)}^{\frac{q r}{r-n}}\right)\|\nabla \mathfrak{u}\|_{L^{q}\left(B_{+}\right)}^{q}\right. \\
& \left.\quad+\frac{R^{q}}{\rho^{* q}}\|\nabla \mathfrak{u}\|_{L^{q}\left(B_{+}\right)}^{q}+\frac{R^{2 q}}{\rho^{* 2 q}}\|\mathfrak{u}\|_{L^{q}\left(B_{+}\right)}^{q}\right)+\varepsilon^{q}\left\|\nabla^{2} \mathfrak{u}\right\|_{L^{q}\left(B_{+}\right)}^{q} .
\end{aligned}
$$

Changing the variables via pullback $\Psi$, we have that

$$
\begin{aligned}
& \left\|\nabla^{2} \widetilde{u}\right\|_{L^{q}\left(\Omega \cap B\left(x_{k}, 2 \varepsilon_{0}\right)\right)}^{q} \\
& \leq C\left(\|\widetilde{f}\|_{L^{q}(\mathcal{N})}^{q}+\mathbf{A}\left(n, r, \rho^{*}, R\right)^{q}\|\nabla \widetilde{u}\|_{L^{q}(\mathcal{N})}^{q}\right. \\
& \left.\quad+\frac{R^{q}}{\rho^{* q}}\|\nabla \widetilde{u}\|_{L^{q}(\mathcal{N})}^{q}+\frac{R^{2 q}}{\rho^{* 2 q}}\|\widetilde{u}\|_{L^{q}(\mathcal{N})}^{q}\right)+\varepsilon^{q}\left\|\nabla^{2} \widetilde{u}\right\|_{L^{q}(\mathcal{N})}^{q} .
\end{aligned}
$$

Summing all estimates with respect to $k$, by the choice $\varepsilon_{0}$ and $\varepsilon$ such that $\Omega_{\varepsilon_{0}} \subset$ $\bigcup_{k} B\left(x_{k}, \varepsilon\right)$ we have that

$$
\begin{aligned}
& \left\|\nabla^{2} \widetilde{u}\right\|_{L^{q}\left(\Omega_{\varepsilon_{0}}\right)}^{q} \\
& \leq C\left(\|\widetilde{f}\|_{L^{q}(\Omega)}^{q}+\mathbf{A}\left(n, r, \rho^{*}, R\right)^{q}\|\nabla \widetilde{u}\|_{L^{q}(\Omega)}^{q}\right. \\
& \left.\quad+\frac{R^{q}}{\rho^{* q}}\|\nabla \widetilde{u}\|_{L^{q}(\Omega)}^{q}+\frac{R^{2 q}}{\rho^{* 2 q}}\|\widetilde{u}\|_{L^{q}(\Omega)}^{q}\right)+\varepsilon^{q}\left\|\nabla^{2} \widetilde{u}\right\|_{L^{q}(\Omega)}^{q} .
\end{aligned}
$$

Finally combining the estimates (3.5) with (3.6), we get

$$
\begin{array}{r}
\left\|\nabla^{2} \widetilde{u}\right\|_{L^{q}(\Omega)} \leq C\left(\|\widetilde{f}\|_{L^{q}(\Omega)}+\mathbf{A}(n, r, \widetilde{\rho}, R)\|\nabla \widetilde{u}\|_{L^{q}(\Omega)}\right. \\
\left.+\frac{R}{\widetilde{\rho}}\|\nabla \widetilde{u}\|_{L^{q}(\Omega)}+\frac{R^{2}}{\widetilde{\rho}^{2}}\|\widetilde{u}\|_{L^{q}(\Omega)}\right),
\end{array}
$$

where $\widetilde{\rho}=\max \left(\rho, \rho^{*}\right)$. Then by converting (3.7) back into the unscaled variables such that $R x \mapsto x$, we obtain

$$
\begin{gathered}
\left\|\nabla^{2} u\right\|_{L^{q}\left(\Omega_{R}\right)} \leq C\left(\|f\|_{L^{q}\left(\Omega_{R}\right)}+R^{-1} \mathbf{A}(n, r, \widetilde{\rho}, R)\|\nabla u\|_{L^{q}\left(\Omega_{R}\right)}\right. \\
\left.+\|\nabla u\|_{L^{q}\left(\Omega_{R}\right)}+\|u\|_{L^{q}\left(\Omega_{R}\right)}\right), \\
A(n, r, \widetilde{\rho}, R)=R\left(\widetilde{\rho}^{-\frac{n}{r}}\|\nabla A\|_{L^{r}\left(\Omega_{R}\right)}+\|\nabla A\|_{\left.L^{(} \Omega_{R}\right)}^{\frac{r}{r-n}}\right) .
\end{gathered}
$$


Using a well-known interpolation inequality (for instance see Theorem 5.2 in [1]) that $\|\nabla v\|_{L^{q}(\Omega)} \leq C\|v\|_{L^{q}(\Omega)}^{\frac{1}{2}}\|v\|_{W^{2, q(\Omega)}}^{\frac{1}{2}}$ for any $v \in W^{2, q}(\Omega)$ and for some constant $C$ independent of $v$. Now by scaling $(u(x) \mapsto u(R x))$ and rescaling $(u(R x) \mapsto u(x))$ with parameter $R>1$ we have

$$
\begin{aligned}
\|\nabla u\|_{L^{q}\left(\Omega_{R}\right)} & \leq C\|u\|_{L^{q}\left(\Omega_{R}\right)}^{\frac{1}{2}}\left(R^{-2}\|u\|_{L^{q}\left(\Omega_{R}\right)}+R^{-1}\|\nabla u\|_{L^{q}\left(\Omega_{R}\right)}+\left\|\nabla^{2} u\right\|_{L^{q}\left(\Omega_{R}\right)}\right)^{\frac{1}{2}} \\
& \leq C\|u\|_{L^{q}\left(\Omega_{R}\right)}^{\frac{1}{2}}\|u\|_{W^{2, q\left(\Omega_{R}\right)}}^{\frac{1}{2}} .
\end{aligned}
$$

Substituting this into (3.8) and applying Young's inequality, we finally conclude the estimate (1.9).

\section{Proof of Theorems 1.2 And 1.3}

Multiplying $u$ to (1.1) and integrating over $\Omega_{R}$, by the ellipticity of $L$ and nonnegativity of $m$ we have

$$
\|\nabla u\|_{L^{2}\left(\Omega_{R}\right)} \leq C\|f\|_{D^{-1}\left(\Omega_{R}\right)} .
$$

Since $f \in L^{\frac{2 n}{n-2}}\left(\Omega_{R}\right)$, Theorem 1.1 holds for $q=q_{0}=\frac{2 n}{n-2}$. Using the Sobolev embedding $D_{0}^{1} \hookrightarrow L^{\frac{2 n}{n-2}}$, we easily get from (4.1)

$$
\|u\|_{L^{q_{0}}\left(\Omega_{R}\right)} \leq C\|f\|_{D^{-1}\left(\Omega_{R}\right)} .
$$

Since the embedding $D_{0}^{1} \hookrightarrow L^{\frac{2 n}{n-2}}$ is invariant with respect to the scaling, the constant $C$ in (4.2) does not depend on $R$. Thus we first get

$$
\|u\|_{W^{2, q_{0}\left(\Omega_{R}\right)}} \leq C\|f\|_{\left(D^{-1} \cap L^{q_{0}}\right)\left(\Omega_{R}\right)}\left(1+\|\nabla A\|_{L^{r}\left(\Omega_{R}\right)}\right)^{\frac{2 r}{r-n}}
$$

If $q_{k}$ is finite, then there holds the embedding $\|v\|_{L^{q_{j+1}(\Omega)}} \leq C\|v\|_{W^{2, q_{j}(\Omega)}}$ for any $0 \leq j \leq k-1$. Here the constant $C$ does not depend on $v$. By scaling invariance of the above embedding for $R>1$ we have

$$
\|u\|_{L^{q_{j+1}\left(\Omega_{R}\right)}} \leq C\|u\|_{W^{2, q_{j}\left(\Omega_{R}\right)}},
$$

where $C$ does not depend on $R$. Thus by induction

$$
\begin{aligned}
& \|u\|_{L^{q_{k}\left(\Omega_{R}\right)}} \leq C\|f\|_{\left(D^{-1} \cap L^{q_{0}} \cap L^{q}\right)\left(\Omega_{R}\right)}\left(1+\|\nabla A\|_{L^{r}\left(\Omega_{R}\right)}\right)^{\frac{2 r k}{r-n}}, \\
& \|u\|_{W^{2, q_{k}\left(\Omega_{R}\right)}} \leq C\|f\|_{\left(D^{-1} \cap L^{q_{0}} \cap L^{q}\right)\left(\Omega_{R}\right)}\left(1+\|\nabla A\|_{L^{r}\left(\Omega_{R}\right)}\right)^{\frac{2 r(k+1)}{r-n}} .
\end{aligned}
$$

Using the embedding (2.5) with $s=q_{k}, t=q, k=2$ and $\Gamma=\Omega$, from the invariance under scaling with parameter $R>1$ and the estimates (4.3) we deduce that

$$
\|u\|_{L^{q}\left(\Omega_{R}\right)} \leq C\|f\|_{\left(D^{-1} \cap L^{q_{0}} \cap L^{q}\right)\left(\Omega_{R}\right)}\left(1+\|\nabla A\|_{L^{r}\left(\Omega_{R}\right)}\right)^{\frac{2 r(k+\delta)}{r-n}} .
$$

Therefore the inequality (1.11) follows from (1.9). 
Now we consider the case $m \geq \underline{m}$. Similarly to the above argument, the integration by parts shows that

$$
\|u\|_{H^{1}\left(\Omega_{R}\right)} \leq C\|f\|_{D^{-1}\left(\Omega_{R}\right)} .
$$

Using the estimate (2.5) with $s=2, \ell=q, k=1$ and the scale invariance with respect to $R>1$, we have for $2 \leq q<\frac{2 n}{n-2}$

$$
\|u\|_{L^{q}\left(\Omega_{R}\right)} \leq C\|u\|_{L^{2}\left(\Omega_{R}\right)}^{1-n(1 / 2-1 / q)}\|u\|_{H^{1}\left(\Omega_{R}\right)}^{n(1 / 2-1 / q)} \leq C\|f\|_{D^{-1}\left(\Omega_{R}\right)} .
$$

Substituting this into (1.9), we obtain the desired estimate (1.12).

From now on we prove Theorem 1.3. Since $L$ is an elliptic operator for small $\varepsilon>0$, it is clear that a unique solution $u$ exists in $\left(W_{0}^{1, q} \cap W^{2, q}\right)\left(\Omega_{R}\right)$ such that $\|\nabla u\|_{L^{2}\left(\Omega_{R}\right)} \leq C\|f\|_{D^{-1}\left(\Omega_{R}\right)}$. For the estimate (1.13), we rewrite the equation $L u=f$ by $L_{B} u=a^{-1}\left(f+\nabla a B \nabla u-\varepsilon L_{E} u\right)$. Since $L_{B}$ is a constant elliptic operator, by the estimate (1.7), we have for some small $\varepsilon$ that

$$
\begin{aligned}
& \left\|\nabla^{2} u\right\|_{L^{2}\left(\Omega_{R}\right)} \\
& \leq C \underline{a}^{-1}\left(\|f\|_{L^{2}\left(\Omega_{R}\right)}+\|\nabla a\|_{L^{r}\left(\Omega_{R}\right)}\|\nabla u\|_{L^{\frac{2 r}{r-2}}\left(\Omega_{R}\right)}\right. \\
& \left.\quad+\varepsilon\left(\|\nabla E\|_{L^{r}\left(\Omega_{R}\right)}\|\nabla u\|_{L^{\frac{2 r}{r-2}}\left(\Omega_{R}\right)}+\Lambda\left\|\nabla^{2} u\right\|_{L^{2}\left(\Omega_{R}\right)}\right)\right) \\
& \leq C\left(\|f\|_{L^{2}\left(\Omega_{R}\right)}+\left(\|\nabla a\|_{L^{r}\left(\Omega_{R}\right)}+\|\nabla E\|_{L^{r}\left(\Omega_{R}\right)}\right)\|\nabla u\|_{L^{\frac{2 r}{r-2}}\left(\Omega_{R}\right)}\right)+\frac{1}{4}\left\|\nabla^{2} u\right\|_{L^{2}\left(\Omega_{R}\right)} .
\end{aligned}
$$

Using the scale invariant estimate (2.5) with $s=2, \ell=\frac{2 r}{r-2}, k=1$, by the fact $2<\frac{2 r}{r-2}<q_{0}$ we have

$$
\begin{aligned}
\|\nabla u\|_{L^{\frac{2 r}{r-2}}} & \leq C\|\nabla u\|_{L^{2}\left(\Omega_{R}\right)}^{1-\frac{n}{r}}\|\nabla u\|_{H^{1}\left(\Omega_{R}\right)}^{\frac{n}{r}} \\
& \leq C\|f\|_{D^{-1}\left(\Omega_{R}\right)}+C\|f\|_{D^{-1}\left(\Omega_{R}\right)}^{1-\frac{n}{r}}\left\|\nabla^{2} u\right\|_{L^{2}\left(\Omega_{R}\right)}^{\frac{n}{r}} .
\end{aligned}
$$

Substituting this into (4.4) and using Young's inequality we obtain

$$
\begin{aligned}
& \left\|\nabla^{2} u\right\|_{L^{2}\left(\Omega_{R}\right)} \\
& \leq C\|f\|_{\left(D^{-1} \cap L^{2}\right)\left(\Omega_{R}\right)}\left(1+\|\nabla a\|_{L^{r}\left(\Omega_{R}\right)}+\|\nabla E\|_{L^{r}\left(\Omega_{R}\right)}\right)^{\frac{r}{r-n}}+\frac{1}{2}\left\|\nabla^{2} u\right\|_{L^{2}\left(\Omega_{R}\right)}
\end{aligned}
$$

and hence

$$
\|\nabla u\|_{H^{1}\left(\Omega_{R}\right)} \leq C\|f\|_{\left(D^{-1} \cap L^{2}\right)\left(\Omega_{R}\right)}\left(1+\|\nabla a\|_{L^{r}\left(\Omega_{R}\right)}+\|\nabla E\|_{L^{r}\left(\Omega_{R}\right)}\right)^{\frac{r}{r-n}} .
$$

On the other hand, it follows from the hypothesis $2 \leq q<q_{0}$ and Lemma 2.4 that

$$
\begin{aligned}
\|\nabla u\|_{L^{q}\left(\Omega_{R}\right)} & \leq\|\nabla u\|_{L^{2}\left(\Omega_{R}\right)}^{1-n\left(\frac{1}{2}-\frac{1}{q}\right)}\|\nabla u\|_{H^{1}\left(\Omega_{R}\right)}^{n\left(\frac{1}{2}-\frac{1}{q}\right)} \\
& \leq C\|f\|_{\left(D^{-1} \cap L^{2}\right)\left(\Omega_{R}\right)}\left(1+\|\nabla a\|_{L^{r}\left(\Omega_{R}\right)}+\|\nabla E\|_{L^{r}\left(\Omega_{R}\right)}\right)^{\frac{r}{r-n}} .
\end{aligned}
$$


Now using the elliptic estimate (1.7) again for any $2 \leq q<q_{0}$, similarly to (4.4) we have

$$
\begin{aligned}
& \left\|\nabla^{2} u\right\|_{L^{q}\left(\Omega_{R}\right)} \\
& \leq C\left(\|f\|_{L^{q}\left(\Omega_{R}\right)}+\left(\|\nabla a\|_{L^{r}\left(\Omega_{R}\right)}+\|\nabla E\|_{L^{r}\left(\Omega_{R}\right)}\right)\|\nabla u\|_{L^{\frac{q r}{r-q}\left(\Omega_{R}\right)}}\right) \\
& +\frac{1}{4}\left\|\nabla^{2} u\right\|_{L^{q}\left(\Omega_{R}\right)} .
\end{aligned}
$$

Since $q<\frac{q r}{r-q}<\frac{n q}{n-q}$ (here $\frac{n q}{n-q}=\infty$ if $q \geq n$ ), an application of Lemma 2.4 yields that

$$
\|\nabla u\|_{L^{\frac{q r}{r-q}\left(\Omega_{R}\right)}} \leq C\|\nabla u\|_{L^{q}\left(\Omega_{R}\right)}^{1-\frac{n}{r}}\|\nabla u\|_{W^{1, q}\left(\Omega_{R}\right)}^{\frac{n}{r}} .
$$

Substituting this into (4.7) and using Young's inequality together with (4.6), we obtain (1.13).

\section{Proof of Theorem 1.4}

Since $f \in D^{-1}(\Omega)$, by the Lax-Milgram theorem, there exists a unique weak solution $u \in D_{0}^{1}(\Omega)$ with $\|\nabla u\|_{L^{2}(\Omega)} \leq C\|f\|_{D^{-1}}$ to the boundary value problem:

$$
L u=f, \quad u=0 \text { on } \partial \Omega, \quad u(x) \rightarrow 0 \text { as }|x| \rightarrow \infty .
$$

Now let $f^{R}$ be a restriction of $f$ to the domain $\widetilde{\Omega}_{R}$ as defined in Remark 3 . Then clearly $f^{R} \in\left(D^{-1} \cap L^{q_{0}} \cap L^{q}\right)\left(\widetilde{\Omega}_{R}\right)$ and the size of its norm is uniform on $R$. From Theorems 1.1 and 1.2 it follows that there exists a unique solution $u^{R} \in\left(W_{0}^{1, q} \cap W^{2, q}\right)\left(\widetilde{\Omega}_{R}\right)$ to the boundary value problem $L u^{R}=f^{R}$ in $\widetilde{\Omega}_{R}$ and $u^{R}=0$ on $\partial \widetilde{\Omega}_{R}$ such that

$$
\begin{aligned}
& \left\|\nabla u^{R}\right\|_{L^{2}\left(\widetilde{\Omega}_{R}\right)} \leq C\left\|f^{R}\right\|_{D^{-1}\left(\widetilde{\Omega}_{R}\right)} \leq C\|f\|_{D^{-1}(\Omega)} \\
& \left\|u^{R}\right\|_{W^{2, q}\left(\widetilde{\Omega}_{R}\right)} \leq C\|f\|_{\left(D^{-1} \cap L^{q_{0}} \cap L^{q}\right)(\Omega)}\left(1+\|\nabla A\|_{L^{r}(\Omega)}\right)^{\frac{2 r(k+1+\delta)}{r-n}}
\end{aligned}
$$

where $\delta=\frac{n}{2}\left(1 / q_{k}-1 / q\right)$. If we extend $u^{R}$ by defining 0 outside $\widetilde{\Omega}_{R}$, then by the weak compactness, there exists a subsequence $u^{R_{j}}$ and $w$ such that $u^{R_{j}} \rightarrow w$ weakly in $\left(D_{0, l o c}^{1} \cap W_{l o c}^{2, q}\right)(\Omega)$ and for any $R$ suitably large

$$
\begin{aligned}
& \|\nabla w\|_{L^{2}\left(\widetilde{\Omega}_{R}\right)} \leq C\|f\|_{D^{-1}(\Omega)}, \\
& \|w\|_{W^{2, q}\left(\widetilde{\Omega}_{R}\right)} \leq C\|f\|_{\left(D^{-1} \cap L^{\left.q_{0} \cap L^{q}\right)(\Omega)}\right.}\left(1+\|\nabla A\|_{L^{r}(\Omega)}\right)^{\frac{2 r(k+1+\delta)}{r-n}} .
\end{aligned}
$$

In view of the weak formulation, $w$ becomes a strong solution satisfying the equations $L w=f$. The uniqueness assertion shows that $u=w$. Therefore the first part of Theorem 1.4 follows. The second and third parts are proved similarly. Details are omitted. 


\section{Application to a parabolic system: Proof of Theorem 1.5}

For sufficiently large $R$, consider the parabolic system

$$
\begin{aligned}
& \varphi^{R} u_{t}^{R}+L u^{R}=F^{R} \text { on }[0, T] \times \widetilde{\Omega}_{R}, \\
& u^{R}(0)=u_{0}^{R} \text { on } \widetilde{\Omega}_{R},
\end{aligned}
$$

where $\varphi^{R}=\varphi+R^{-2}$ on $\widetilde{\Omega}_{R}, F^{R}=\psi^{R} F$ for some nonnegative cut-off function $\psi^{R}$ supported in $\widetilde{\Omega}_{R}$ with $\left|\nabla \psi^{R}\right| \leq 100 R^{-1}, u_{0}^{R} \in\left(H_{0}^{1} \cap H^{2}\right)\left(\widetilde{\Omega}_{R}\right)$ is a solution to the boundary value problem

$$
\left(\left.L\right|_{t=0}\right) u_{0}^{R}=F^{R}(0)-\sqrt{\varphi^{R}} g \text { on } \widetilde{\Omega}_{R} .
$$

Then the unique existence of smooth solution $u^{R}$ follows readily from the standard argument of parabolic system ${ }^{1}$.

Now we consider some a priori estimates for the solution $u^{R}$. Multiplying $u_{t}$ to (6.1) and integrating over $\widetilde{\Omega}_{R}$, we get

$$
\int_{\tilde{\Omega}_{R}} \varphi^{R}\left|u_{t}^{R}\right|^{2} d x+\sum_{i, j, \alpha, \beta} \int_{\tilde{\Omega}_{R}} A_{i j}^{\alpha \beta} \partial_{i} u_{t}^{R, \alpha} \partial_{j} u^{R, \beta} d x=<F^{R}, u_{t}^{R}>.
$$

The symmetry of $A(1.18)$ shows that

$$
\begin{gathered}
\int_{\widetilde{\Omega}_{R}} \varphi^{R}\left|u_{t}^{R}\right|^{2} d x+\frac{1}{2} \frac{d}{d t} \sum_{i, j, \alpha, \beta} \int_{\widetilde{\Omega}_{R}} A_{i j}^{\alpha \beta} \partial_{i} u^{R, \alpha} \partial_{j} u^{R, \beta} d x \\
=<F^{R}, u_{t}^{R}>+\frac{1}{2} \sum_{i, j, \alpha, \beta} \int_{\widetilde{\Omega}_{R}}\left(A_{i j}^{\alpha \beta}\right)_{t} \partial_{i} u^{R, \alpha} \partial_{j} u^{R, \beta} d x .
\end{gathered}
$$

Integrating over $[0, t]$, we have from the ellipticity of $A$ and Hölder inequality that

$$
\begin{aligned}
\int_{0}^{t}\left\|\sqrt{\varphi^{R}} u_{t}\right\|_{L^{2}\left(\widetilde{\Omega}_{R}\right)}^{2} d s+\frac{\lambda}{2}\left\|\nabla u^{R}\right\|_{L^{2}\left(\widetilde{\Omega}_{R}\right)}^{2} \\
\leq C\left\|\nabla u_{0}^{R}\right\|_{L^{2}\left(\tilde{\Omega}_{R}\right)}^{2}+\int_{0}^{t}\left(\varepsilon^{-1}\left\|F^{R}\right\|_{D^{-1}\left(\tilde{\Omega}_{R}\right)}^{2}+\varepsilon\left\|\nabla u_{t}\right\|_{L^{2}\left(\tilde{\Omega}_{R}\right)}^{2}\right. \\
\left.+C\left\|A_{t}\right\|_{L^{6}(\Omega)}\left\|\nabla u^{R}\right\|_{L^{2}\left(\tilde{\Omega}_{R}\right)}\left\|\nabla u^{R}\right\|_{L^{3}\left(\tilde{\Omega}_{R}\right)}\right) d s .
\end{aligned}
$$

Hereafter we will use $C$ as a generic constant independent of $R$.

Now after taking $\partial_{t}$ to (6.1), if we multiply $u_{t}$ on both sides and integrate them over $\widetilde{\Omega}_{R}$, then we obtain from the ellipticity of $L$ that

$$
\begin{aligned}
& \frac{1}{2} \frac{d}{d t} \int_{\tilde{\Omega}_{R}} \varphi^{R}\left|u_{t}^{R}\right|^{2} d x+\lambda\left\|\nabla u_{t}^{R}\right\|_{L^{2}\left(\tilde{\Omega}_{R}\right)}^{2} \\
& \quad \leq<F_{t}^{R}, u_{t}^{R}>-\sum_{i, j, \alpha, \beta} \int_{\tilde{\Omega}_{R}}\left(A_{i j}^{\alpha \beta}\right)_{t} \partial_{i} u_{t}^{R, \alpha} \partial_{j} u^{R, \beta} d x
\end{aligned}
$$

\footnotetext{
${ }^{1}$ One can use the Galerkin approximation as in [8].
} 
Since $\varphi^{R} u_{t}^{R}(0)=F^{R}(0)-\left(\left.L\right|_{t=0}\right) u_{0}^{R}=\sqrt{\varphi^{R}} g$, one can show that

$$
\frac{1}{2}\left\|\sqrt{\varphi^{R}} u_{t}^{R}(0)\right\|_{L^{2}\left(\widetilde{\Omega}_{R}\right)} \leq \frac{1}{2}\|g\|_{L^{2}(\Omega)} .
$$

Hence integrating (6.4) over $[0, t]$, we have by Hölder's and Young's inequalities that

$$
\begin{aligned}
& \left\|\sqrt{\varphi^{R}} u_{t}^{R}\right\|_{L^{2}\left(\widetilde{\Omega}_{R}\right)}^{2}+\lambda \int_{0}^{t}\left\|\nabla u_{t}^{R}\right\|_{L^{2}\left(\widetilde{\Omega}_{R}\right)}^{2} d s \\
& \leq\|g\|_{L^{2}(\Omega)}^{2}+C \int_{0}^{t}\left(\left\|F_{t}^{R}\right\|_{D^{-1}\left(\widetilde{\Omega}_{R}\right)}^{2}+\left\|\nabla u^{R}\right\|_{L^{3}\left(\widetilde{\Omega}_{R}\right)}^{2}\right) d s .
\end{aligned}
$$

Combining (6.3) and (6.5) for $\varepsilon=\frac{\lambda}{2}$, we have for any $t \in[0, T]$

$$
\begin{aligned}
& \left\|\sqrt{\varphi^{R}} u_{t}^{R}\right\|_{L^{2}\left(\widetilde{\Omega}_{R}\right)}^{2}+\frac{\lambda}{2}\left\|\nabla u^{R}(t)\right\|_{L^{2}\left(\widetilde{\Omega}_{R}\right)}^{2}+\frac{\lambda}{2} \int_{0}^{t}\left\|\nabla u_{t}^{R}\right\|_{L^{2}\left(\widetilde{\Omega}_{R}\right)}^{2} d s \\
& \leq C\left\|\nabla u_{0}^{R}\right\|_{L^{2}\left(\widetilde{\Omega}_{R}\right)}^{2}+\|g\|_{L^{2}(\Omega)}^{2}+C \int_{0}^{t}\left(\left\|F^{R}\right\|_{D^{-1}\left(\widetilde{\Omega}_{R}\right)}^{2}+\left\|F_{t}^{R}\right\|_{D^{-1}\left(\widetilde{\Omega}_{R}\right)}^{2}\right) d s \\
& \quad+C \int_{0}^{t}\left(\varepsilon^{-1}+\left\|A_{t}\right\|_{L^{6}(\Omega)}^{2}\right)\left\|\nabla u^{R}\right\|_{L^{2}\left(\widetilde{\Omega}_{R}\right)}^{2} d s+\varepsilon \int_{0}^{t}\left\|\nabla u^{R}\right\|_{L^{6}\left(\widetilde{\Omega}_{R}\right)}^{2} d s .
\end{aligned}
$$

Here for the last integral we used the Hölder inequality that $\left\|\nabla u^{R}\right\|_{L^{3}\left(\widetilde{\Omega}_{R}\right)} \leq$ $\left\|\nabla u^{R}\right\|_{L^{2}\left(\widetilde{\Omega}_{R}\right)}^{\frac{1}{2}}\left\|\nabla u^{R}\right\|_{L^{6}\left(\widetilde{\Omega}_{R}\right)}^{\frac{1}{2}}$ and Young's inequality.

By the elliptic estimate (1.11) with $k=0, q_{0}=6$ and $r=6$ (hence $\delta=0$ ),

$$
\begin{aligned}
& \left\|\nabla u^{R}\right\|_{W^{1,6}\left(\widetilde{\Omega}_{R}\right)} \\
& \leq C\left(\left\|F^{R}\right\|_{\left(D^{-1} \cap L^{6}\right)\left(\widetilde{\Omega}_{R}\right)}+\left\|\varphi^{R} u_{t}^{R}\right\|_{\left(D^{-1} \cap L^{6}\right)\left(\widetilde{\Omega}_{R}\right)}\right)\left(1+\|\nabla A\|_{L^{6}(\Omega)}\right)^{4} .
\end{aligned}
$$

From the choice of $\psi^{R}$ we observe that

$$
\left\|F^{R}\right\|_{\left(D^{-1} \cap L^{6}\right)\left(\widetilde{\Omega}_{R}\right)} \leq C\|F\|_{\left(D^{-1} \cap L^{6}\right)(\Omega)} .
$$

Moreover, from the assumption $\varphi \in L^{\frac{3}{2}}(\Omega)$ that for any $v \in D_{0}^{1}\left(\widetilde{\Omega}_{R}\right)$

$$
\begin{aligned}
<\varphi^{R} u_{t}^{R}, v> & =<\left(\varphi+R^{-2}\right) u_{t}^{R}, v> \\
& \leq\left(\|\varphi\|_{L^{\frac{3}{2}}(\Omega)}+C R^{-2+2}\right)\left\|u_{t}^{R}\right\|_{L^{6}\left(\widetilde{\Omega}_{R}\right)}\|v\|_{L^{6}\left(\widetilde{\Omega}_{R}\right)} \\
& \leq C\left(1+\|\varphi\|_{L^{\frac{3}{2}(\Omega)}}\right)\left\|\nabla u_{t}^{R}\right\|_{L^{2}\left(\widetilde{\Omega}_{R}\right)}\|\nabla v\|_{L^{2}\left(\widetilde{\Omega}_{R}\right)} .
\end{aligned}
$$

Hence

$$
\left\|\varphi^{R} u_{t}^{R}\right\|_{\left(D^{-1} \cap L^{6}\right)\left(\widetilde{\Omega}_{R}\right)} \leq C\left(1+\|\varphi\|_{\left(L^{\frac{3}{2}} \cap L^{\infty}\right)(\Omega)}\right)\left\|\nabla u_{t}^{R}\right\|_{L^{2}\left(\widetilde{\Omega}_{R}\right)} .
$$

Now let us define a constant $C_{0}$ as

$$
\begin{aligned}
C_{0} \equiv 1 & +\left\|\nabla u_{0}\right\|_{L^{2}(\Omega)}+\|g\|_{L^{2}(\Omega)}+\|\varphi\|_{\left(L^{\frac{3}{2}} \cap L^{\infty}\right)(\Omega)}+\sup _{[0, T]}\left(\|\nabla A\|_{L^{2}(\Omega)}+\left\|A_{t}\right\|_{L^{6}(\Omega)}\right) \\
& +\|F\|_{L^{2}\left(0, T ;\left(D^{-1} \cap L^{6}\right)(\Omega)\right)}+\left\|F_{t}\right\|_{L^{2}\left(0, T ; D^{-1}(\Omega)\right)} .
\end{aligned}
$$


Then substituting the above two estimates into (6.7), we have

$$
\left\|\nabla u^{R}\right\|_{W^{1,6}\left(\widetilde{\Omega}_{R}\right)} \leq C C_{0}^{4}\left(\|F\|_{\left(D^{-1} \cap L^{6}\right)(\Omega)}+\left\|\nabla u_{t}^{R}\right\|_{L^{2}\left(\widetilde{\Omega}_{R}\right)}\right) .
$$

Plugging this into (6.6), we have for any $t \in[0, T]$

$$
\begin{aligned}
& \left\|\sqrt{\varphi^{R}} u_{t}^{R}\right\|_{L^{2}\left(\widetilde{\Omega}_{R}\right)}^{2}+\frac{\lambda}{2}\left\|\nabla u^{R}(t)\right\|_{L^{2}\left(\widetilde{\Omega}_{R}\right)}^{2}+\frac{\lambda}{2} \int_{0}^{t}\left\|\nabla u_{t}^{R}\right\|_{L^{2}\left(\widetilde{\Omega}_{R}\right)}^{2} d s \\
& \leq C C_{0}^{10}+C\left(\varepsilon^{-1}+C_{0}^{2}\right) \int_{0}^{t}\left\|\nabla u^{R}\right\|_{L^{2}\left(\widetilde{\Omega}_{R}\right)}^{2} d s+\varepsilon C C_{0}^{8} \int_{0}^{t}\left\|\nabla u_{t}^{R}\right\|_{L^{2}\left(\widetilde{\Omega}_{R}\right)}^{2} d s .
\end{aligned}
$$

If we choose $\varepsilon$ with $\varepsilon C C_{0}^{8} \leq \frac{\lambda}{4}$, then by Gronwall's inequality we get for any $t \in[0, T]$

$$
\left\|\sqrt{\varphi^{R}} u_{t}^{R}(t)\right\|_{L^{2}\left(\widetilde{\Omega}_{R}\right)}^{2}+\left\|\nabla u^{R}(t)\right\|_{L^{2}\left(\widetilde{\Omega}_{R}\right)}^{2}+\int_{0}^{t}\left\|\nabla u_{t}^{R}\right\|_{L^{2}\left(\widetilde{\Omega}_{R}\right)}^{2} d s \leq C_{T},
$$

where $C_{T}$ is a constant depending on $C, C_{0}$ and $T$. From (6.8) it follows that

$$
\int_{0}^{T}\left\|\nabla u^{R}\right\|_{W^{1,6}\left(\widetilde{\Omega}_{R}\right)}^{2} d t \leq C_{T}
$$

Now extending $\varphi^{R}, u_{0}^{R}, F^{R}$ and $g$ by defining zero outside $\widetilde{\Omega}_{R}$ to $\Omega$, it is an easy matter to show $u_{0}^{R}$ is a weak solution of (6.2) replaced by $\Omega$ and $u_{0}^{R} \rightarrow u_{0}$ in $D_{0}^{1}(\Omega)$. Similarly, extending the solution $u^{R}$ by defining zero outside $\widetilde{\Omega}_{R}$, by the above a priori estimates $(6.9)$ and $(6.10)$ we deduce that there exists a subsequence $u^{R_{j}}$ converging in weak or weak* sense to a solution $u \in L^{\infty}\left(0, T ; D_{0}^{1}\right)$ of $(1.17)$ satisfying that $\nabla u \in L^{2}\left(0, T ; W^{1,6}(\Omega)\right), u_{t} \in L^{2}\left(0, T ; D_{0}^{1}(\Omega)\right)$. The uniqueness follows immediately from the weak formulation of (1.17). This completes the proof of the theorem.

\section{REFERENCES}

[1] R. A. Adams and J.J.F. Fournier, Sobolev Spaces, Pure and Applied Mathematics Series 140, Elsevier, Oxford, 2003.

[2] S. Agmon, A. Douglis and L. Nirenberg, Estimates near the boundary for solutions of elliptic partial differential equations satisfying general boundary conditions. I., Comm. Pure Appl. Math. 12 (1959), 623-727.

[3] S. Agmon, A. Douglis and L. Nirenberg, Estimates near the boundary for solutions of elliptic partial differential equations satisfying general boundary conditions. II., Comm. Pure Appl. Math., 17 (1964), 35-92.

[4] Y. Cho, H. J. Choe and H. Kim, Unique solvability of the initial boundary value problems for compressible viscous fluids, J. Math. Pures Appl., 83 (2004), 243-275.

[5] Y. Cho and H. Kim, Existence results for viscous polytropic fluids with vacuum, J. Differential Equations 228 (2006), 377-411.

[6] Y. Cho and H. Kim, Existence result for heat-conducting viscous incompressible fluids with vacuum, to appear in J. Korean Math. Soc. 
[7] A. Douglis and L. Nirenberg, Interior estimates for elliptic systems of partial differential equations, Comm. Pure Appl. Math., 8 (1955), 503-530.

[8] L. C. Evans, Partial Differential Equations, American Mathematical Society, 1998.

[9] G. P. Galdi, An Introduction to the Mathematical Theory of the Navier-Stokes Equations, in "Springer Tracts in Natural Philosophy 38," Springer-Verlag, New York, 1994.

[10] M. Giaquinta, An Introduction to the Regularity Theory for Nonlinear Elliptic Systems, Birkhäuser, Basel, 1993.

[11] D. Gilbarg and N. S. Trudinger, Elliptic Partial Differential Equations of Second Order, Springer-Verlag, New York, 1983.

[12] M. Giaquinta and G. Modica, Nonlinear systems of the type of the stationary Navier-Stokes system, J. Reine Angew. Math., 330 (1982), 173-214.

[13] P. -L. Lions, Mathematical Topics in Fluid Mechanics Vol. 1, 2, Clarendon Press, Oxford, 1998.

[14] C. B. Morrey, Jr., Multiple Integrals in the Calculus of Variations, Springer-Verlag, Berlin and New York, 1966.

[15] L. Nirenberg, Remarks on strongly elliptic partial differential equations, Comm. Pure Appl. Math., 8 (1955), 648-674.

[16] L. Nirenberg, Estimates and existence of solutions of elliptic equations, Comm. Pure Appl. Math., 9 (1956), 509-530.

[17] L. Nirenberg, On elliptic partial differential equations, Ann. Scuola Norm. Pisa, 13 (1959), 115-162.

[18] E. M. Stein, Harmonic Analysis, Princeton, New Jersey, 1993.

Department of Mathematics, Chonbuk University, Jeonju 561-756, Republic of Korea

E-mail address: changocho@chonbuk.ac.kr

Department of Mathematics, Hokkaido University, Sapporo 060-0810, Japan

E-mail address: ozawa@math.sci.hokudai.ac.jp

Department of Mathematics, Postech, Pohang 790-784, Republic of Korea

E-mail address: shim@postech.ac.kr 